OPEN ACCESS

Edited by:

Wei Liu,

Chongqing University, China

Reviewed by:

Jinyang Fan,

Chongqing University, China

Guimin Zhang,

China University of Mining and

Technology, China

Yintong Guo,

Institute of Rock and Soil Mechanics

(CAS), China

*Correspondence:

Yunan $\mathrm{Li}$

liyunan@cug.edu.cn

Anming Wang

wam992001@163.com

Specialty section: This article was submitted to

Geohazards and Georisks,

a section of the journal

Frontiers in Earth Science

Received: 03 November 2021 Accepted: 02 December 2021

Published: 13 January 2022

Citation:

He J, Li Y, Jin Y, Wang A, Zhang Y, Jia J, Song H and Liang D (2022) Study on Mechanical Problems of Complex

Rock Mass by Composite Material Micromechanics Methods: $A$

Literature Review.

Front. Earth Sci. 9:808161.

doi: 10.3389/feart.2021.808161

\section{Study on Mechanical Problems of Complex Rock Mass by Composite Material Micromechanics Methods: A Literature Review}

\author{
Junzhao $\mathrm{He}^{1,2}$, Yunan $\mathrm{Li}^{1 *}$, Yuling $\mathrm{Jin}^{3}$, Anming Wang ${ }^{4 *}$, Yumin $\mathrm{Zhang}^{4}$, Jinchao Jia ${ }^{4}$, \\ Hei Song ${ }^{2}$ and Dong Liang ${ }^{2}$
}

${ }^{1}$ China University of Geosciences, Wuhan, China, ${ }^{2}$ Henan Geological and Mineral Resources Construction Engineering (Group) Co. Ltd, Zhengzhou, China, ${ }^{3}$ nstitute of Geodesy and Geomatics, Henan Bureau of Geology and Mineral Resources, Zhengzhou, China, ${ }^{4}$ North China University of Water Conservancy and Electric Power, Zhengzhou, China

The mechanical analysis of complex rock mass is a difficult problem, which often occurs in scientific research and practical engineering. Many achievements have been made in the study of rock mass composite problems by composite material micromechanics method, but it has not been well summarized so far. This paper summarizes in detail the research status of complex rock mass problems by composite material micromechanics method at home and abroad, including the application of the Eshelby equivalent inclusion theory and self-consistent model in rock mass composite problems, and the application of the homogenization method in jointed rock mass and other rock mass composite problems such as anchored rock mass, layered rock mass, and salt rock mass with impurities. It is proposed that the structural similarity and mechanical analysis similarity should be satisfied when the composite material micromechanics method is used to study the complex rock mass. Finally, the problems that need to be further studied are put forward. The research results provide a valuable reference for the study of complex rock mass by the composite material micromechanics method.

Keywords: complex rock mass, composite material micromechanics method, Eshelby equivalent inclusion theory, self-consistent model, homogenization method

\section{INTRODUCTION}

As a very complex structure, the rock contains defects such as the holes, stratification, schistosity, joints, weak structural planes, and interlayer. In addition, micro-structures, micro-cracks, or multiphase inclusions are also formed in the microstructure, and the mineral crystals that make up rocks also bear a variety of forms. The spatial distribution, geometric size, and internal filling type of these defects have a great influence on the physical and mechanical properties of rock materials (Chen and Li, 1992). Therefore, the study of the evolution law of cracks or defects under various external factors such as force, temperature, and moisture has important significance for some special rock mass structures such as fissure rock mass, jointed rock mass, weak interlayer rock mass, and impurity rock mass.

In the early days, the elastic mechanics method was used to analyze the rock materials. Subsequently, plastic mechanics, fracture damage mechanics, explosive mechanics, statistical mechanics, computational mechanics, and computer technology are increasingly applied for rock 
mechanics. However, rock is a kind of complex geological material, and the discipline of rock mechanics and engineering should not only develop its theories, technologies, and methods from other basic disciplines but also carry out multidisciplinary cross-collaboration (She and Peng, 2014). The composite mechanics is a science of specializing in mechanical behavior under different material combinations based on theoretical mechanics, material mechanics, elasticity mechanics, plasticity mechanics, etc.

Based on the idea of applying composite material mechanical methods to study the rock mechanics, as early as 1956, Einstein (Einstein, 1956) was apparently the first to attempt such work, followed by Hashin (Hashin and Shtrikman, 1963). After that, a great number of scholars (Salamon, 1968; Eshelby, 1957; Li et al., 2006a; Abou-ChakraGue' et al., 2008) have studied the mechanical problems of complex rock mass by composite material micromechanics methods. In 1979, Chinese Professor Wang Jingtao (Wang, 1979) pointed out that it is meaningful to deal with the problems of rock mechanics by using the basic principles and composite material micromechanics methods, and it can more realistically reflect the mechanical properties of rock mass. In 1990, Professor Zhao Pinglao (Zhao and Zeng, 1990) believed that studying rock mechanics by the composite material mechanics methods would be a favorite new idea to promote the rapid development of rock mechanics.

This article mainly summarizes the application of composite material micromechanics methods in the field of rock mechanics, and analyzes and discusses the relevant problems in order to provide relevant researchers with a more comprehensive understanding of the research status in this field and promote the further application of composite material micromechanics methods to the research of geotechnical engineering problems.

\section{BASIC THEORY OF COMPOSITE MATERIAL MECHANICS AND ITS APPLICATION IN ROCK MECHANICS}

The theoretical research methods of mechanical properties of composite materials are mainly divided into macromechanical methods and micro-mechanical methods (Qin and Yang, 2006). In the macro-mechanics theory of composites, the composites are regarded as macrohomogeneous media, and the internal reinforcing phase and matrix are regarded as a whole without considering the interaction between internal components, and the micromechanics is to establish the quantitative relationship among the macro properties, composition properties, and microstructure of composites (Du and Wang, 1998).

As early as 1957, Eshelby (Eshelby, 1957; Eshelby, 1959) put forward Eshelby's equivalent inclusion theory based on a large number of studies. Subsequently, more mature micro-mechanics theories have been put forward, such as the Mori Tanaka method (Mori and Tanaka, 1973), self consistent theory (Hill, 1965a; Budiansky, 1973), and generalized self consistent theory (Christensen and Lo, 1979).
The homogenization method (Benssousan et al., 1978; Cioranescu and Paulin, 1979; Sanchez-Palencia, 1980) developed in the 1970s is used as an alternative method to find the effective characteristics of equivalent homogenized materials. The representative volume element method, the multi-scale progressive homogenization method, and the homogenization method based on inclusion theory are the main contents of the homogenization method.

\section{Eshelby Equivalent Inclusion Theory and Its Application}

Eshelby uses Green's function method of three-dimensional elastic problems to obtain the explicit solution of the ellipsoidal inclusion problem, and also analyzes the elastic stress field around the inclusion (Eshelby, 1957; Eshelby, 1959). More research work has been carried out in the field of rock mechanics. On the one hand, scholars used Eshelby's equivalent inclusion theory to study the application of rock macro-equivalent elastic modulus ( $\mathrm{Hu}$ et al., 2013; Xiao and Huang, 2013; Nguyen et al., 2016), rock rheology, and reinforcement engineering (Xiao and Huang, 2013). At the same time, the change of the rock meso-structure is studied by Eshelby inclusion theory and meso-damage mechanics (Bai, 2016).

Pensee (Vincent and Kondo, 2003) and Zhu (Zhu et al., 2008) combined Eshelby's inclusion theory and energy method to obtain the damage model of brittle materials. Zhu (2006) used the Eshelby inclusion problem solution to construct the thermodynamic framework of the micromechanical damage model, and by considering the elastic strain $\left(\varepsilon^{m}\right)$ of matrix and the inelastic strain $\left(\varepsilon^{c}\right)$ caused by crack, the general form of representative volume element free energy is obtained, as shown in Eq. 1:

$$
W=\frac{1}{2}\left(\varepsilon-\varepsilon^{c}\right): c^{m}:\left(\varepsilon-\varepsilon^{c}\right)+\frac{1}{2} \varepsilon^{c}: c^{b}: \varepsilon^{c},
$$

where : $C^{m}$ is the matrix modulus, which is the fourth-order effective elastic tensor.

Chen et al. (2011) established the meso-damage mechanics model of rock under the condition of thermal mechanical coupling by using the M-T method and Taylor method.

The above is the case of meso-damage mechanics studied by inclusion theory. Within the framework of macro-damage mechanics, Weilide (Lide et al., 2004a; Lide et al., 2004b) used the Eshelby equivalent inclusion method to establish the elastic-plastic constitutive relationship of rock mass considering the plastic deformation of damaged rock. However, because the Eshelby theoretical solution is not applicable to the case of large inclusion proportion, the model simulation results are quite different from the test results in the later stage.

\section{Self-Consistent Model and Its Application}

The basic idea of the self-consistent method is to obtain the average strain in the inclusion phase from the self-consistent model under uniform boundary conditions, so as to obtain the 


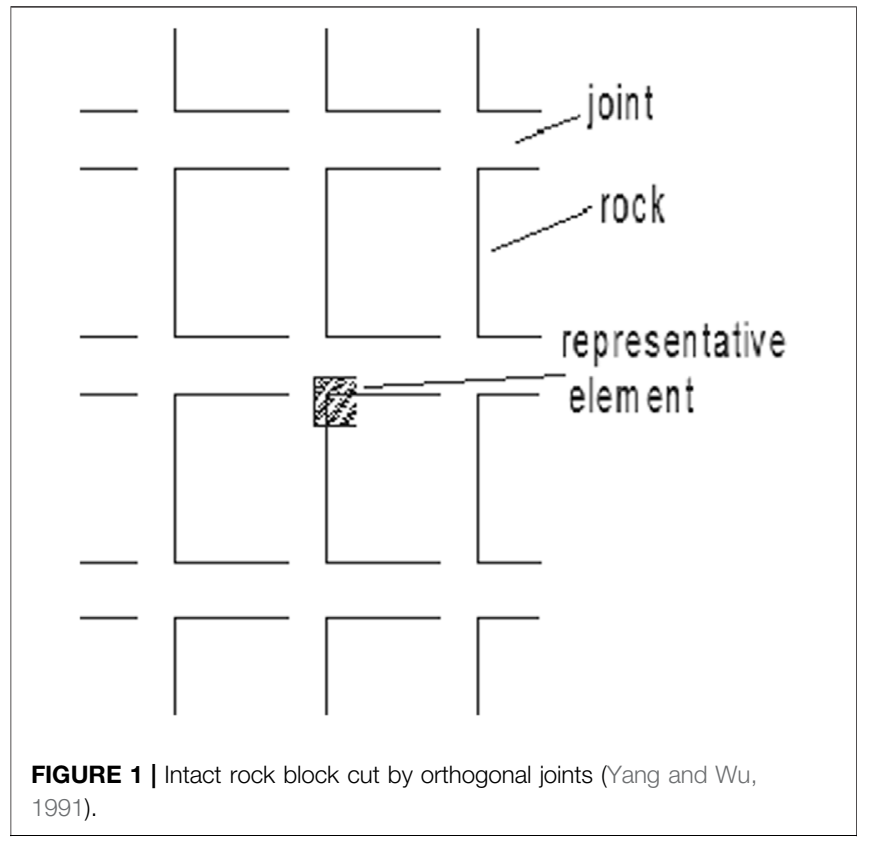

effective elastic stiffness tensor. In view of the defects in the application of the self-consistent method to the calculation of equivalent modulus of multiphase (inclusion) composites (Zheng et al., 2010), Kerner (Kerner, 1956) proposed a generalized autonomous model. The prediction results of the generalized self-consistent model are more reasonable than those of the selfconsistent model (Christensen, 1990). In general, the application of the self-consistent method in rock mechanics is not much. In order to consider the influence of the interaction between microcracks in rock on rock damage, Wen Jianhua and Zhou Cuiying (Wen et al., 2011) studied the damage effect of rock materials based on the generalized self-consistent theory, and established a damage constitutive model reflecting the development and change of rock micro-cracks under load. Wang et al. (2015) developed a self-consistent model for the elastic contact of rough surfaces to account for the asperity interaction through Boussinseq's solution.

\section{The Homogenization Method and Its Application}

The homogenization theory can be applied in many areas of physics and engineering, having finely heterogeneous continuous media, like heat transfer or fluid flow in porous media, or, for example, electromagnetism in composites (Hassani and Hinton, 1998). The homogenization method has also been widely used in the field of rock mechanics, such as joint rock mass, anchored rock mass, and layered rock mass.

\section{Jointed Rock Mass}

Joints have a profound impact on the mechanical properties of rock mass structures. The purpose of studying the mechanical properties of jointed rock mass is to study its macro rock mass properties. For a single jointed rock mass, the joint model can be directly established. For example, Goodman joint element (Goodman et al., 1968) can be easily introduced into numerical analysis. However, the problems of joint set and discontinuity plane are often encountered in practical rock engineering. When analyzing this kind of rock mass, it is very difficult to establish a separate joint element for numerical analysis (Zhang et al., 2012).

Therefore, scholars equalize jointed rock mass into generalized composite materials, regard joints as matrix materials in composite materials, and regard rock mass as an inclusion material in composite materials. Appropriate representative units are selected to equivalent jointed rock into homogeneous rock materials. Typical representative units are shown in Figures 1, 2. In representative units, the constitutive relations of rock mass and joints are given. The macroscopic equivalent mechanical parameters and constitutive model of jointed rock mass can be obtained by using the coordination conditions between macro and meso stress-strain and the mesomechanical method [Zhang $\mathrm{Wu}$ et al. (Zhang and Zhang, 1987); Yang Haitian et al. (Yang and Wu, 1991)], such as the elastic constants and joint occurrence parameters of jointed material and complete rock mass (Zhang and Zhang, 1987), and the composite creep model of jointed rock mass (Yang and Wu, 1991; Maghous et al., 20212021).

It should be noted that there are many forms of joints in practical engineering. Researchers also use the composite meso homogenization method to obtain the macro equivalent constitutive models and parameters of these joints, such as stepped jointed rock mass (Singh, 1973); unidirectional, bidirectional, and multidirectional jointed rock mass (Zhang and Zhang, 1987); oblique jointed rock mass (Niu and Yang, 2007a); and layered jointed rock mass (Huang et al., 2010).

In another case, scholars assume that jointed rock mass has meso-periodic characteristics. First, according to the principle of

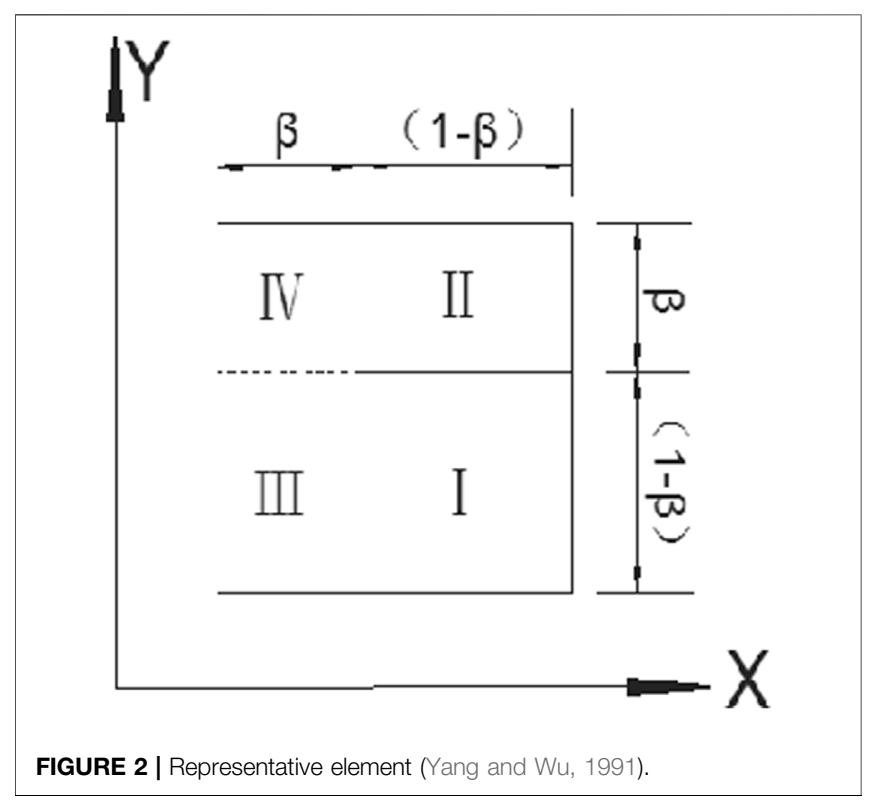




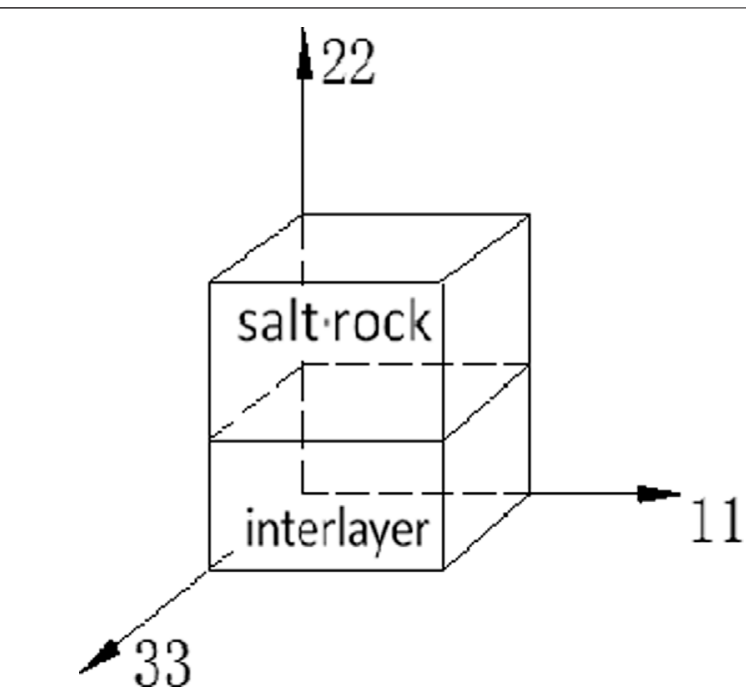

FIGURE 3 | Representative volume element for interbedded salt rock (Wang et al., 2009).

virtual displacement within the range of linear elasticity (Niu and Yang, 2007b; Ren and Xu, 2008), as shown in Eq. 2:

$$
\int_{\Omega} E_{i j k l} \frac{\partial \mu_{k}^{\varepsilon}}{\partial \chi_{i}} \frac{\partial v_{i}}{\partial \chi_{j}} d \Omega=\int_{\Omega} f_{i}^{\varepsilon} \nu_{i} d \Omega+\int_{\Gamma_{t}} t_{i} \nu_{i} d \Gamma,
$$

where $f_{i}^{\varepsilon}$ is the volume force, $t_{i}$ represents the external load acting on the boundary, $\Gamma_{t}, \mu^{\varepsilon}$ is the real displacement, and $v$ is any virtual displacement satisfying the boundary conditions, $i, j, k \in\{1,2,3\}$.

Then, the displacement and stress of a point in the macrostructure are expanded into a small parameter progressive expansion about the meso-structure scale. Combined with the perturbation technology, a series of control equations are obtained. According to these equations, the equivalent material parameters of homogenized jointed rock mass can be solved, as shown in Eq. 3, so as to convert the meso-heterogeneous jointed rock mass into homogeneous material (Ren and $\mathrm{Xu}, 2008$ ).

$$
E_{i j k l}^{H}=\frac{1}{|Y|} \int_{Y}\left(E_{i j k l}-E_{i j p m} \frac{\partial \chi_{p}^{k l}}{\partial y_{m}}\right) d Y .
$$

\section{Anchored Rock Mass}

The mechanical and homogenization methods for composite materials have also been applied to anchored rock mass. The rock mass support system was regarded as a kind of composite materials composed of rock mass (matrix), anchor (reinforcing material), and mortar (bonding material) (Li et al., 2006b), and then the micromechanical elastic constant calculation model for rock mass was established by using composite material mechanical methods. As a further study, the layered rock mass-anchor support system (Li et al., 2006a)-was regarded as a kind of unidirectional composite reinforcement material composed of layered rock mass (matrix material), anchor rod (fiber material), and mortar (carrier material). The relationship between the mechanical properties of the equivalent composite material and each component was quantitatively analyzed.

\section{Layered Rock Mass}

The layered rock mass mentioned here refers to the interbedded rock mass, which is composed of at least two lithologic units with different engineering behaviors and properties. This layered rock mass often presents the structure of alternating layers of hard rock and soft rock with different thicknesses (Zhang et al., 2021). Because this layered rock mass shows high anisotropy and heterogeneity, it is very important to determine the mechanical properties of layered rock mass for the selection of eigenvalues for design and stability analysis in such engineering construction (Liu et al., 2019; Zhang et al., 2019; Liu et al., 2020a).

In the experimental study, layered composite rock samples were often used to carry out the relevant tests (Liang et al. (Liang et al., 2007)). It is clear that complex samples have more complex mechanical parameters or strength characteristics than a single rock sample. The homogenization method is often used for the heterogeneous medium when establishing the constitutive model; that is, the rock mass of various thicknesses is generalized as an equivalent homogeneous medium by using the representative elements. The complex representation element, as shown in Figure 3, is commonly found, and it can be taken as plane strain problems or spatial problems. Of course, the correct mixing rate is chosen according to the structural characteristics of the rock strata, such as the strain coordination principle and the multiphase mixture principle (Fan et al., 1999; Haj-Ali and Muliana, 2003; Haj-Ali and Muliana, 2004). The mixing rate is the bridge between the microscopic volume and the macroscopic volume for the homogenization method, by which the equivalent elasticity, elastic-plastic, and viscous constitutive model of the layered rock mass can be finally obtained. For example, the macroequivalent elastic, elasto-plastic, and non-linear creep constitutive models of interbedded salt rock mass were established by the Yang Chunhe research group (Chun-he and Yin-ping, 2005; Li and Yang, 2006; Wang et al., 2009). Zhao Pinglao (Zhao and Zeng, 1990) has studied the constitutive relations of layered rock mass by means of composite material mechanical methods. Liu Kaoxue et al. (Liu, 1990) have established the viscoelastic model of interbedded and jointed rock mass.

Two-Step Homogenization Method and Its Application In recent years, the two-step homogenization method has been applied to solve the multi-scale problem of heterogeneous materials (Nguyen et al., 2011; Shen et al., 2012; Bikong et al., 2015; Zhao et al., 2019). WQ Shen and JF Shao (Shen et al., 2012; Zhao et al., 2019) have regarded clay rock as a complex of linear elastic mineral particles embedded in the pore clay matrix, and the clay matrix was considered to be made up of the solid phase and spherical micropores. As shown in Figure 4, the clay solid phase and spherical micro-pores are subjected to the first homogenization on the microscopic scale, and the macroscopic homogeneous stress-strain relationship of the clay matrix is obtained. On the basis of the first homogenization, the second homogenization of clay matrix and mineral particles is carried out on the microscopic 


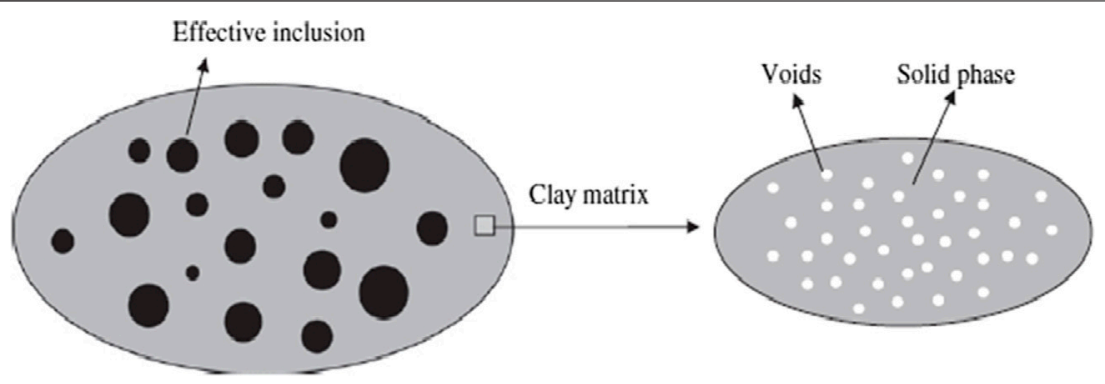

FIGURE 4 | Basic principle program of the two-step homogenization method (Shen et al., 2012).

scale, and the micromechanical model of clay rock is established to evaluate its macroscopic plastic properties.

However, the application of the homogenization method and the establishment of representative elements are always greatly related to the structural characteristics of rock mass. Unlike the jointed rock mass, the isotropic porous rock materials or geomaterials are, in the microstructure scale, composed of nearly spherical grains surrounded by a matrix. Such kinds of materials are encountered in geo-mechanics, such as concretes and some rocks (limestone, ironore, clay rock, etc.). Because this material has the particles in the matrix, the composite material mechanical methods are used well in the homogenization process, such as the Mori Tanaka Scheme (Mori and Tanaka, 1973), Hill's incremental method (Hill, 1965a;Hill, 1965b), and J.F. Shao method (Shen et al., 2012; Bikong et al., 2015; Zhao et al., 2019).

\section{STUDY ON OTHER COMPOSITE GEOTECHNICAL PROBLEMS WITH COMPOSITE MATERIAL MICROMECHANICAL PRINCIPLES}

Composite subgrade and composite soil are common treatment ways for subgrade in geotechnical engineering. It is usually composed of two or more kinds of component materials: one is the soil (matrix) of continuous distribution and the other is a pile or geo-synthetics (reinforcements) surrounded by the soil. Because of the non-uniform structure of composite soil, it is difficult to understand its nature. Ye Guanbao (Ye et al., 2002) proposed a micro-mechanical view of composite subgrade where the core task is to establish a quantitative relationship between the macroscopic performance of composite subgrade and its component performance and its meso-structure, and reveal the response law and essence of composite subgrade under certain working conditions.

Based on the micromechanical homogenization method, many scholars ( Ye et al., 2002; Wang et al., 2002; Lu, 2013) predict the equivalent elastic parameters of pile-soil composite foundation. In other similar studies, Ren et al. (2009) considered the geo-grid and soil as generalized composite materials and established a numerical calculation model to predict the elastic equivalent constitutive parameters.

\section{STUDY ON MECHANICAL PROBLEMS OF IMPURITY SALT ROCK MASS BY COMPOSITE MATERIAL MECHANICAL PRINCIPLE}

Impurity salt rock mass belongs to the concept of rock mass. In addition to pure salt rock, it also contains other common symbiotic mineral components, such as gypsum, anhydrite, glauberite, and other impurities (Ding, 2010). Impurities have a great impact on the overall mechanical properties of impurity salt rock mass (Liu et al., 2020b).

By the light of the composite material mechanical principle, the impurity salt rock mass was considered as the particlereinforced composite material. In addition, the targeted research into the impurity salt rock mass by composite material micromechanical principles may present two types of mechanical analysis models to evaluate the properties of the impurity salt rock mass.

On the one hand, the impurity salt rock mass is regarded as a homogeneous medium, and the continuum constitutive model is deduced with the continuous stress-strain field. From the microscopic viewpoint, the impurity salt rock mass is a nonuniform material composed of different impurities and salt layers. However, from the macroscopic viewpoint, it should be regarded as a uniform material. Therefore, the representative elements can be used for impurity salt rock mass, and the stress-strain relationship of each component of the representative elements can be used to establish the macroscopic equivalent stress-strain relationship of the impurity salt rock mass. The purpose of the micro-mechanical study on composite materials is to establish the quantitative relationship among the macroscopic composite properties, the component properties, and micro-structure. The impurity salt rock mass is considered as a particlereinforced composite material, and the macroscopic and micro-mechanical properties of the impurity salt rock mass can be analyzed by the composite material micromechanical principles.

On the other hand, the impurity salt rock mass itself has obvious structural features. It is a kind of multiphase material (salt rock matrix, impurity-enhanced phase, and interfacial phase). Due to the difference in mechanical properties among the different phases, the various phases will interact with each 
other under external load. The impurity salt rock mass is regarded as an inhomogeneous medium, and the micromechanical finite element method of composite materials, with consideration of different microstructure and composition, can be used to study the internal micro-stress field, displacement field, and overall mechanical properties of the impurity salt rock mass, in terms of the different contents, sizes, shapes, and mechanical properties of impurities. Furthermore, the properties and damage of impurity salt rock mass depend on the properties of salt rock compositions and impurities as well as the micro-structure characteristics such as impurity volume, impurity fraction, impurity shape, impurity size, impurity distribution, and interface phase features. Therefore, the key to revealing the effect of the microscopic characteristics of impurity salt rock mass on its performance depends on how to forecast and analyze its performance and reveal its nature of damage and destruction by micromechanical method.

\section{DISCUSSION ON THE KEY PROBLEMS OF ROCK MECHANICS BY COMPOSITE MATERIAL MICROMECHANICAL METHODS}

\section{Similarity of Structural Composition Between Complex Rock Mass and Composite Materials}

According to the research literature works above, the authors believe that the similarity in the structural composition must exist between rock mass and composite materials when studying rock mechanics issues by composite material mechanical methods.

In the composite materials faculty, composite materials are defined as new materials composed of two or more components. Essentially, the different components were optimized by the most suitable microscopic structure to produce composite materials in order to meet the practical needs of different industries.

As the research object of geotechnical engineering, the natural rock mass is itself a kind of natural geological material. As the literature (Peng, 2010) describes, the difference between the rock mass and other materials mainly lies in the structure, and the material structure is closely related to its scale. Rock is a nonhomogeneous material composed of particles, voids, fissures, and cement. Heterogeneity has a significant effect on the strength and deformation of rock. It can be seen that the idea on rock is just the opposite of that on composite material, but with consistent essence.

In terms of material composition, the composite model (Liu et al., 2011) of the composite material is divided into two types: macro-composite and micro-composite. Macro-composite refers mainly to the overlap between two or more layers of different materials (also known as lamination), such as aluminum alloy sheet and carbon fiber or glass fiber composite sheet stack. A microscopic composite, such as a particle-reinforced, fiberreinforced, or polymer-based composite material, means that one or more fine materials are uniformly dispersed in another continuous material. Therefore, as mentioned in the literature (Chen and Li, 1992), rock is a kind of a complex material which can be considered to contain two components: the matrix and the defects or micro-cracks in the matrix. In addition, there are also rock mass with cements, impurity particles, and weak interlayer, which are commonly referred to as soft and hard interbedded rock mass, interbedded salt rock mass, or impurity salt rock mass. Obviously, from the composition viewpoint, the complex structure of rock mass is also very similar to that of composite materials. Figure 5 shows the micro-structural model of twophase composite materials, which are very similar to those of common geotechnical engineering objects such as columnar jointed rock mass, composite foundation, interbedded rock mass, and impurity salt rock mass.

\section{Similarity in Mechanical Analysis Between Complex Rock Mass and Composite Materials}

Fan et al. (1995) considered that any material capable of two levels of analysis and calculation can be called composite material. Composite material mechanics is a two-level mechanics theory, which is embodied in the establishment of the conversion between two levels of mechanics, namely, between micromechanics and macro-mechanics. In addition, the mechanical properties and damage laws of composite materials depend on the component properties and micro-structure features such as volume fraction, distribution, shape, and interfacial properties of the enhanced phase. It can be seen that the mechanical analysis of composite materials focuses on the influence of microstructural features on the macroscopic properties from the qualitative viewpoint and on the relationship between microscopic mechanics and macroscopic mechanics from the quantitative viewpoint. The macroscopic stress-strain relationship of the composite is deduced by the stress-strain relationship, the volume fraction of microscopic materials, and Eq. 4 (Belayachi et al., 2012).

$$
\overline{\sigma_{i j}}=\frac{1}{V} \int_{V} \sigma_{i j} d V \quad \overline{\varepsilon_{i j}}=\frac{1}{V} \int_{V} \varepsilon_{i j} d V,
$$

where $\overline{\sigma_{i j}}$ and $\overline{\varepsilon_{i j}}$ are the macroscopic average components of the stresses and strains over the whole volume $\mathrm{V}$ of the unit cell, respectively.

From the viewpoint of rock mass mechanics, Ji et al. (2006) believed that the difference in rock types lies mainly in the differences in the volume fraction of rock-forming minerals. As long as the physical and mechanical properties and volume fraction of the main rock-forming minerals are determined, the physical and mechanical properties of any type of composite multi-mineral rock can be calculated by using the correct mixing law. Therefore, it is the key to establishing the correct mixing law. Obviously, this view is consistent with the transition relationship between micromechanics and macroscopic mechanics in the quantitative analysis of composite materials. In addition, Gaofeng and Wei (2005) compared composite materials with rock blocks. The similarity between composite materials and rock 


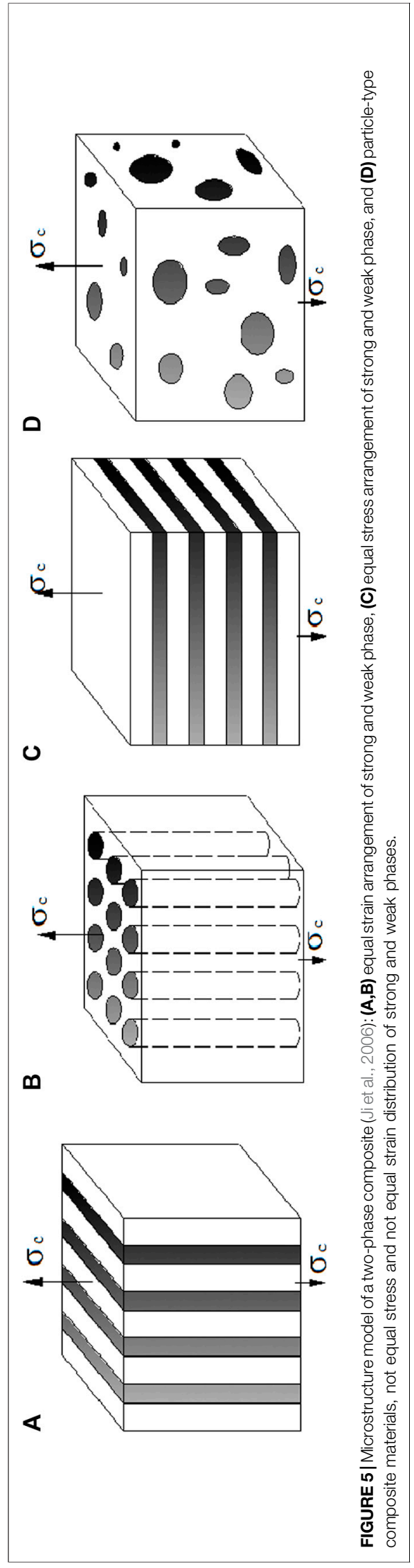

blocks is that there is a clear interface between the two materials, and the no-embedding criterion is satisfied at the interface. The difference is that the displacement of the composite material is continuous at the interface, with the existence of tensile stress and compressive stress, but the rock blocks, when contacting, are only under compressive pressure with continuous displacement, without tensile stress, and detach under tension.

Bolt support systems can be regarded as special composite materials (Li et al., 2006a). From the point of view of composite material, a bolt is a reinforcement material, and rock mass is the matrix material in a composite material. From the point of view of mechanics, bolt reinforcement of surrounding rock improves the mechanical properties of rock mass. Similarly, salt rock mass containing impurities can be regarded as particle reinforced composites (Wang et al., 2011), in which impurities are regarded as the "reinforcing phase" in composites and salt rock is regarded as the "matrix phase." From the point of view of the mechanical mechanism, the "reinforcement and creep inhibition" of impurities on the salt rock layer is essentially consistent with the "reinforcement and creep inhibition" of particles on the matrix.

\section{DISCUSSION AND OUTLOOK}

In summary, scholars in the world have carried out fruitful research work in the study on rock mechanics using composite material micromechanics methods. The authors believe that the following aspects should be mainly concerned in the future study:

\section{Mechanical Properties of Interface}

The combination of the rocks in the underground rock mass is quite different at the boundary (Tan et al., 1994). The calculated stress, strain, and strength at the boundary with cohesion are obviously different from those without cohesion. One of the most important problems in composite materials composed of grains surrounded by a matrix with a strong contrast of stiffness between them, such as concrete materials and also rock-composite materials, is the interphase zone or interfacial transition zone (ITZ) between grain and matrix. Therefore, more rock mechanical experiments and microscopic experiments should be carried out to understand the mechanical properties and bonding action of the interface; study the interface stress transfer, interface damage, and slip mechanisms; and explore the effect of interface performance on the macro-mechanical and micro-mechanical properties.

\section{Macro-Failure Criteria}

Due to the physical combination and structural characteristics, the composite materials are totally different from homogeneous materials in the failure mode. Uniform materials fail macroscopically and evenly as a whole, but composite materials fail in the weakest section among the reinforcement, matrix, and interface. The failure form and law of composite materials are related 
not only to the stress state but also to the component performance and combination situation.

Similar to composite materials, relative displacement and stress transfer will occur among the phases of the complex rock mass when the external load changes. Thus, the mechanical response, deformation, and destruction of complex rock mass result from the micro-effects among internal components and represent the macroscopic group behavior. Therefore, the macroscopic deformation and failure mechanisms should be researched in terms of the micro-structure level, which is also the major problem in studying the macroscopic failure criterion of rock complex mechanics.

\section{Multiphase Complex of Geotechnical Engineering}

Guo (2000) proposed the concept of multiphase materials which have a wide range of material composition and microstructure, with clear micro-structure direction, organizational diversity, anisotropy, and scale effect, compared to the two-phase composite materials. Thus, the multiphase materials demonstrate more complicated mechanical behavior. For example, the anchoring rock and soil in geotechnical complexes can be regarded as three-phase composite materials composed of the bolt (cable), cement slurry, and rock (soil), which can be further studied by means of composite material micromechanical methods.

\section{Combination Between Digital Image Processing Technique and Finite Element Method}

As known by all, the microscopic behavior detection methods, such as scanning electron microscopy (SEM), transmission electron microscopy (TEM), electron backscatter diffraction (EBSD), neutron diffraction, and digital image technology, cannot provide microscopic information about stress and strain, and the pure finite element model cannot reflect the real microscopic characteristics and microscopic compositions of materials.

Next, the rock samples can be sliced by using digital image processing technology (Yue et al., 2004; Kang et al., 2007; Yan et al., 2017) in combination with the meso-experimental research method of composite material, and the real twodimensional microstructure of the rock samples can be obtained through the digital image of a scanning electron microscope. Then the microstructure is imported into the finite element software for meshing, which can more truly

\section{REFERENCES}

Abou-ChakraGue', A., Cormery, F., Shao, J.-F., and Kondo, D. (2008). A Micromechanical Model of Elastoplastic and Damage Behavior of a Cohesive Geomaterial. Int. J. Sol. Structures45, 1406-1429. doi:10.1016/ j.ijsolstr.2007.09.025 reflect the shape and distribution of particles in rock mass and obtain more accurate calculation results.

\section{CONCLUSION}

It is of great practical significance to study the mechanical problems of complex rock mass by using composite material micromechanics methods. This article summarizes in detail the application of the composite micromechanics method, Eshelby inclusion theory, and self-consistent model, especially the homogenization method, in the combination of jointed rock mass, anchored rock mass, layered rock mass, and complex salt rock mass. On this basis, the author believes that when the composite material micromechanics methods are used to study the composite problem of rock mass, the complex rock mass should be similar to the composite material in structure and consistent with the composite material in mechanical analysis. In the next step, the mechanical properties of the composite rock mass interface, failure criteria, multiphase geotechnical composite problems, and the combination of digital image processing technology and finite element method can be studied by using or drawing lessons from the mechanical method of composite materials.

\section{AUTHOR CONTRIBUTIONS}

$\mathrm{JH}$ has made contributions to the conception of the study, the geological characteristics of the rock mass structure, anchored rock mass and the composite foundation, and revised, discussed, and wrote the part of the first draft. YL contributed to the conception of this study. YJ, HS, and DL provided technical advice on rock mass structure and geology. AW wrote the part of the first draft. AW, YZ, and JJ polished the first draft. All authors contributed to the revision, reading, and approval of the first draft.

\section{FUNDING}

This research was financed by "Intelligent monitoring, simulation, control, and early warning of urban geological disasters based on geological big data" (u1711266), a key project jointly funded by NSFC and Guangdong Province. Jia Tao, Wu Jiaqi, Hao Shun, and Xing Jiangpeng et al. took part in the study, and sincere gratitude is expressed to them.

Bai, J. (2016). Study of Equivalent Elastic Modulus of Sand Gravel Soil with Eshelby Tensor and Mori-Tanaka Equivalent Method. Shanxi Architecture42 (8), 81-83. (in Chinese). doi:10.13719/j.cnki.cn14-1279/ tu.2012.01.143

Belayachi, N., Do, D. P., and Hoxha, D. (2012). A Note on the Numerical Homogenisation of the Mechanical Behaviour of an Argillaceous Rock. Comput. Geotechnics41, 70-78. doi:10.1016/j.compgeo.2011.11.007 
Benssousan, A., Lions, J. L., and Papanicoulau, G. (1978). Asymptotic Analysis for Periodic Structures. North Holland: Amesterdam.

Bikong, C., Hoxha, D., and Shao, J. F. (2015). A Micro-macro Model for Time-dependent Behavior of Clayey Rocks Due to Anisotropic Propagation of Microcracks. Int. J. Plasticity69, 73-88. doi:10.1016/ j.ijplas.2015.02.001

Budiansky, B. (1973). On the Elastic Moduli of Some Heterogeneous Materials. J.Mech.Phys.Solids.13, 223-227. doi:10.1016/0022-5096(65)90011-6

Chen, L., and Li, C. (1992). Constitutive Relation of Rock. Prog. Mech.22 (2), 173-181. (in Chinese). doi:10.13245/j.hust.1990.05.009

Chen, Y., Li, D., Rong, G., Jiang, Q., and Zhou, C. (2011). A Micromechanical Modelfor Damage and thermal Conductivity of Brittle Rocks. Chin. J. Rock Mech. Eng. 30 (10), 1959-1969. (in Chinese).

Christensen, R. M. (1990). A Critical Evaluation for a Class of Micro-mechanics Models. J. Mech. Phys. Sol.38 (3), 379-404. doi:10.1016/0022-5096(90)90005-o

Christensen, R. M., and Lo, K. H. (1979). Solutions for Effective Shear Properties in Three Phase Sphere and cylinder Models. J. Mech. Phys. Sol.27 (4), 315-330. doi:10.1016/0022-5096(79)90032-2

Chun-he, Y., and Yin-ping, L. I. (2005). The Expanded Cosserat Medium Constitutive Model for Laminated Salt Rock. Chin. J. Rock Mech. Eng.24 (23), 4226-4232. (in Chinese). doi:10.3321/j.issn:10006915.2005.23.005

Cioranescu, D., and Paulin, J. S. J. (1979). Homogenization in Open Sets with Holes. J. Math. Aanal Appl.71, 590-607. doi:10.1016/0022-247x(79)90211-7

Ding, G. (2010). Salt Hole Underground Gas Storage. Beijing: Petroleum Industry Press. (in Chinese).

Du, S., and Wang, B. (1998). Composite Materials Mesoscopic Mechanics. Beijing: Science Press. (in Chinese).

Einstein, A. (1956). Investigations on the Theory of Brownian Motion. New York): Dover Pubns., 36-62.

Eshelby, J. D. (1957). The Determination of the Elastic Field of an Ellipsoidal Inclusion and Related Problems. Proc. R. Soc. Scr. A.241, 376-396. doi:10.1098/ rspa.1957.0133

Eshelby, J. D. (1959). The Elastic Field outside an Ellipsoidal Inclusion. Proc. Roy. Soc. London.252, 561-569doi:10.1098/rspa.1959.0173

Fan, F., Wang, Z., Xing, J., and Huang, X. (1995). Research on Several Basic Issues of Composite Material Mechanics. J. south China Univ. Technol. (natural Sci. edition)23 (4), 1-6. doi:10.3321/j.issn:1000-565X.1995.04.001

Fan, J., Gao, Z., and Liu, T. (1999). The Micro/macro Mechanic Analysis of PD3 Pearlitic Steel with Layered Microstructures. J. Chongqing Univ.22 (5), 37-46. (in Chinese). doi:10.11835/j.issn.1000-582X.1999.05.008

Gaofeng, W., and Wei, F. (2005). Three-dimensional Numerical Manifold Method and its Application in Composite Materials. J. Appl. Mech.22 (3), 351-356. (in Chinese). doi:10.3969/j.issn.1000-4939.2005.03.005

Goodman, R. E., Taylor, R. L., and Brekke, T. L.(1968). A Model for the Mechanics of Jointed Rock . J. Soil Mech. Found. Div. 94(3):637-659. doi:10.1061/ jsfeaq.0001133

Guo, J. (2000). From Composite Materials to Multiphase Materials. Chin. J. Mater. Res. 14 (2), 123-126. (in Chinese). doi:10.3321/j.issn:1005-3093.2000.02.002

Haj-Ali, R. M., and Muliana, A. H. (2003). A Micromechanical Constitutive Framework for the Nonlinear Viscoelastic Behavior of Pultruded Composite Materials. Int. J. Sol. Structures40, 1037-1057. doi:10.1016/s0020-7683(02) 00663-7

Haj-Ali, R. M., and Muliana, A. H. (2004). A Multi-Scale Constitutive Formulation for the Nonlinear Viscoelastic Analysis of Laminated Composite Materials and Structures. Int. J. Sol. Structures41, 3461-3490. doi:10.1016/ j.ijsolstr.2004.02.008

Hashin, Z., and Shtrikman, S. (1963). A Variational Approach to the Theory of the Elastic Behaviour of Multiphase Materials. J. Mech. Phys. Sol.11, 127-140. doi:10.1016/0022-5096(63)90060-7

Hassani, B., and Hinton, E. (1998). A Review of Homogenization and Topology Optimization I-Homogenization Theory for media with Periodic Structure. Comput. Structures69, 707-717. doi:10.1016/s00457949(98)00131-x

Hill, R. (1965a). A Self-Consistent Mechanics of Composite Materials. J. Mech. Phys. Sol.13, 213-222. doi:10.1016/0022-5096(65)90010-4

Hill, R. (1965b). Continuum Micro-mechanics of Elastoplastic Polycrystals. J. Mech. Phys. Sol.13, 89-101. doi:10.1016/0022-5096(65)90023-2
$\mathrm{Hu}, \mathrm{M} ., \mathrm{Xu}, \mathrm{G}$. , and Hu, S. (2013). Study of Equivalent Elastic Modulus of Sand Gravel Soil with Eshelby Tensor and Mori-Tanaka Equivalent Method, 34, 1437-1442. (in Chinese).

Huang, S., Xu, J., Ding, X., and Aiqing, W. (2010). Study of Layered Rock Mass Composite Model Based on Characteristic of Structural Plane and its Application. Chin. J. Rock Mech. Eng.29 (4), 743-756. (in Chinese).

Ji, S., Wang, Q., Xia, B., and Xu, Z. (2006). Generalized Mixed Law and its Application in Geomaterial Rheology. Acta Petrologica Sinica22 (7), 2067-2080.

Kang, J., Ososkov, Y., Embury, J., and Wilkinson, D. (2007). Digital Image Correlation Studies for Microscopic Strain Distribution and Damage in Dual Phase Steels. Scripta Materialia56 (11), 999-1002. doi:10.1016/ j.scriptamat.2007.01.031

Kerner, E. H. (1956). The Elastic and Thermoelastic Properties of Composite media. Proc. Phys.69, 801-808. doi:10.1088/0370-1301/69/8/305

Li, X., Song, G., Chen, X., and Yang, W. (2006). Research on the Composite Material Mechanical Properties of Anchoring Rock Mass. J. Wuhan Univ. Tech.28 (4), 79-82. (in Chinese). doi:10.3321/j.issn:1671-4431.2006.04.023

Li, X., Wang, T., Song, G., Yunhua, C., and Hang Chengliang, Z. (2006). Study on Composite Anchoring Theory and Numerical Simulation Test on Layered Rocks. Chin. J. Rock Mech. Eng.25 (2), 3654-3660. (in Chinese). doi:10.3321/ j.issn:1000-6915.2006.z2.050

Li, Y., and Yang, C. (2006). Three-dimensional Expanded Cosserat Medium Constitutive Model for Laminated Salt Rock. Rock Soil Mech.27 (4), 509-513. (in Chinese). doi:10.16285/j.rsm.2006.04.001

Liang, W., Yang, C., Zhao, Y., Dusseault, M. B., and Liu, J. (2007). Experimental Investigation of Mechanical Properties of Bedded Salt Rock. Int. J. Rock Mech. Mining Sci.44, 400-411. doi:10.1016/j.ijrmms.2006.09.007

Lide, W., Xu, W., Yang, C., and Yang, S. (2004). Study on Elastic Constitutive Model of Rock with Statistucal Damage[J]. Chin. J. Rock Mech. Eng.23 (12), 1971-1975. (in Chinese). doi:10.3321/j.issn:1000-6915.2004.12.00

Lide, W., Yang, C., and Xu, W. (2004). Study on Statistical Seepage Model and Statistical Damage Constitutive Model of Rock. Rock Soil Mech.25 (10), 1527-1536. (in Chinese). doi:10.16285/j.rsm.2004.10.00

Liu, K. (1990). Viscoelastic Model of Layered Rock Mass and Jointed Rock Mass. J. Shaanxi Inst. Machinery6, 292-307. (in Chinese). doi:10.19322/ j.cnki.issn.1006-4710.1990.04.009

Liu, W., Yu, Y., and Gao, L. (2011). Composites. Harbin: Harbin Institute of Technology Press. (in Chinese).

Liu, W., Zhang, X., Fan, J., Zuo, J., Zhang, Z., and Chen, J. (2020). Study on the Mechanical Properties of Man-Made Salt Rock Samples with Impurities. J. Nat. Gas Sci. Eng.84, 103683. doi:10.1016/j.jngse.2020.103683

Liu, W., Zhang, Z., Chen, J., Fan, J., Jiang, D., Jjk, D., et al. (2019). Physical Simulation of Construction and Control of Two Butted-Well Horizontal Cavern Energy Storage Using Large Molded Rock Salt Specimens. Energy185, 682-694. doi:10.1016/j.energy.2019.07.014

Liu, W., Zhang, Z., Fan, J., Jiang, D., Li, Z., and Chen, J. (2020). Research on Gas Leakage and Collapse in the Cavern Roof of Underground Natural Gas Storage in Thinly Bedded Salt Rocks. J. Energ. Storage31, 101669. doi:10.1016/ j.est.2020.101669

Lu, Y. (2013). Application of Asymptotic Homogenization Method in Settlement Calculation of Biaxial Reinforced concrete Composite Foundation. Master's thesis. Xiangtan: Xiangtan University. (in Chinese).

Maghous, S., Barros de Aguiar, C., and Rossi, R. (20212021). Micromechanical Approach to Effective Viscoelastic Behavior of Jointed Rocks. Int. J. Rock Mech. Mining Sci.139, 104581. doi:10.1016/j.ijrmms.2020.104581

Mori, T., and Tanaka, K. (1973). Average Stress in Matrix and Average Elastic Energy of Materials with Misfitting Inclusions. Acta Metallurgica21, 571-574. doi:10.1016/0001-6160(73)90064-3

Nguyen, B. N., Hou, Z., Bacon, D. H., Murray, C. J., and White, M. D. (2016) Three-dimensional Modeling of the Reactive Transport of $\mathrm{CO} 2$ and its Impact on Geomechanical Properties of Reservoir Rocks and Seals. Int. J. Greenhouse Gas Control.46, 100-115. doi:10.1016/j.ijggc.2016.01.004

Nguyen, N. B., Giraud, A., and Grgic, D. (2011). A Composite Sphere Assemblage Model for Porous Oolitic Rocks. Int. J. Rock Mech. Mining Sci.48, 909-921. doi:10.1016/j.ijrmms.2011.05.003

Niu, B., and Yang, H. (2007). Homogenization-based Investigation on Constitutive Relationship of Oblique Joint Rock. Chin. J. Geotechnical Eng.29 (5), 773-778. 
Niu, B., and Yang, H. (2007). Study on Composite Constitutive Relation of Skew Jointed Rock Mass Based on Homogenization Method. J. Geotechnical Eng.29 (5), 773-778. (in Chinese). doi:10.3321/j.issn:1000-4548.2007.05.024

Peng, C. (2010). "Rock Mass Generalized Multi-Layer Structure Model," in Doctoral Dissertation (Hangzhou: Zhejiang University). (in Chinese)s.

Qin, Q., and Yang, Q. (2006). Macro-meso Theory of Multi-Field Coupling Behavior of Non-uniform Material. Beijing: Higher Education Press. (in Chinese).

Ren, Q., and $\mathrm{Xu}, \mathrm{W}$. (2008). Homogenization_based Method for Predicting Effective Elastic Properties of Jointed Rock. Eng. Mech.25 (4), 75-92.

Ren, Y., Yang, H., Niu, B., and Yan, J. (2009). Study on Equivalent Constitutive Relation of Geogrid Based on Homogenization Method. Dalian Univ. Tech.49 (3), 317-321. (in Chinese).

Salamon, M. D. G. (1968). Elastic Moduli of a Stratified Rock Mass. Int. J. Rock Mech. \&Mining Sci.5, 519-527. doi:10.1016/0148-9062(68)90039-9

Sanchez-Palencia, E. (1980). Non-homogenous media and Vibration Theory, 127. New York, NY: Lecture Notes in Physics.

She, S., and Peng, L. (2014). Some Progress and Challenges of Rock Engineering in China. Chin. J. Rock Mech. Eng.33 (3), 433-457. (in Chinese). doi:10.13722/ j.cnki.jrme.2014.03.001

Shen, W. Q., Shao, J. F., Kondo, D., and Gatmiri, B. (2012). A Micro-macro Model for Clayey Rocks with a Plastic Compressible Porous Matrix. Int. J. Plasticity36, 64-85. doi:10.1016/j.ijplas.2012.03.006

Singh, B. (1973). Continuum Characterization of Jointed Rock Masses. Int. J. Rock Mech. Mining Sci. Geomechanics Abstr.10, 311-335. doi:10.1016/0148-9062(73)90041-7

Tan, X., Xian, X., Zheng, D., and Zhao, Y. (1994). Composite Rock Mechanics Theory and Application. Beijing: Coal Industry Press. (in Chinese).

Vincent, P., and Kondo, D. (2003). Micromechanics of Anisotropic Brittle Damage: Comparative Analysis between a Stress Based and a Strain Based Formulation. Mech. Mater.35, 747-761. doi:10.1016/s0167-6636(02)00203-x

Wang, A., Yang, C., Chen, J., Huang, Z., and Li, Y. (2009). Nonlinear Creep Constitutive Model of Layered Rock Salt. J. Rock Mech. Eng.28 (1), 2708-2714. (in Chinese). doi:10.3321/j.issn:1000-6915.2009.z1.018

Wang, A., Yang, C., Chen, J., Zhang, X., and Huang, Z. (2011). "Composite Material Method for Studying Mechanical and Deformation Properties of Layered Salt Rock," in Proceeding of the 3rd International Conference on Heterogeneous Material Mechanics, 421-424. (in Chinese).

Wang, G. F., Long, J. M., and Feng, X. Q. (2015). A Self-Consistent Model for the Elastic Contact of Rough Surfaces. Acta Mech.226, 285-293. doi:10.1007/ s00707-014-1177-2

Wang, J. (1979). Propagation of Cylindrical Wave in Stratified Rock Mass. Rock Soil Mech. (2), 73-80. (in Chinese). doi:10.16285/j.rsm1979.02.005

Wang, J. G., Leung, C. F., and Ichikawa, Y. (2002). A Simplified Homogenisation Method for Composite Soils. Comput. Geotechnics29, 477-500. doi:10.1016/ s0266-352x(02)00004-6

Wen, J., Zhou, C., and Peng, M. (2011). On Statistical Damage Constitutive Model for Rock Based on General Self-Consistent Theory. Subgrade Eng. (4), 1-3. (in Chinese). doi:10.3969/j.issn.1003-8825.2011.04.001

Xiao, C., and Huang, S. (2013). The Application of Eshelby's Theory on Rock Mechanics Discussion, 34, 1437-1442. (in Chinese).5

Yan, L., Meng, Q.-x., Xu, W.-y., Wang, H.-1., Zhang, Q., Zhang, J.-c., et al. (2017). A Numerical Method for Analyzing the Permeability of Heterogeneous Geomaterials Based on Digital Image Processing. J. Zhejiang Univ. Sci. A.18 (2), 124-137. (in Chinese). doi:10.1631/jzus.A1500335

Yang, H., and Wu, R. (1991). A Composite Creep Model of Biorthogonal Joint Jointed Rock Mass. Dalian Univ. Tech.31 (3), 267-273. (in Chinese).

Ye, G., Lan, H., Xu, C., Chen, J., Bai, H., and Gao, Y. (2002). Microscopic Mechanical Properties of Composite Foundation. Rock Soil Mech.23, 48-51. (in Chinese). doi:10.3969/j.issn.1000-7598.2002.z1.01310.16285/j.rsm.2002.s1.041
Yue, Z., Chen, S., Zheng, H., and Tan, G. (2004). Digital Image Proceeding Based on Finite Element Method for Geomaterials. Chin. J. rock Mech. Eng.23 (6), 889-897. (in Chinese). doi:10.3321/j.issn:10006915.2006.10.002

Zhang, H., Zhu, J., Liu, Y., Xu, B., and Wang, X. (2012). Strength Properties of Jointed Rock Masses Based on the Homogenization Method. Acta Mechanica Solida Sinica25 (2), 177-185. (in Chinese). doi:10.1016/ so894-9166(12)60018-4

Zhang, W., and Zhang, X. (1987). Elastic Model of the Rock Mass. Chin. J. Geotechnical Eng.9 (4), 33-44. (in Chinese). doi:10.3321/j.issn:10004548.1987.04.004

Zhang, X., Liu, W., Jiang, D., Qiao, W., Liu, E., Zhang, N., et al. (2021). Investigation on the Influences of Interlayer Contents on Stability and Usability of Energy Storage Caverns in Bedded Rock Salt. Energy231, 120968. doi:10.1016/j.energy.2021.120968

Zhang, Z., Jiang, D., Liu, W., Chen, J., Li, E., Fan, J., et al. (2019). Study on the Mechanism of Roof Collapse and Leakage of Horizontal Cavern in Thinly Bedded Salt Rocks. Environ. Earth Sci.78 (10), 292. doi:10.1007/s12665-019$8292-2$

Zhao, L.-Y., Shao, J.-F., Zhu, Q.-Z., Liu, Z.-B., and Yurtdas, I. (2019). Homogenization of Rock-like Materials with Plastic Matrix Based on an Incremental Variational Principle. Int. J. Plasticity123, 145-164. doi:10.1016/ j.ijplas.2019.07.015

Zhao, P., and Zeng, Y. (1990). Composite Model of Layered Rock Mass. J. Lanzhou Univ. (Natural Sci. Edition)26 (2), 114-118. (in Chinese). doi:10.13885/ j.issn.0455-2059.1990.02.020

Zheng, X., Zheng, X., and Duan, L. (2010). Progress of Multiscale Methods in the Mechanical Analysis of Composites[J]. Adv. Mech.40 (1), 41-56. (in Chinese). doi:10.6052/1000-0992-2010-1-J2008-104

Zhu, Q. (2006). Applications of Homogenization Approaches to 3D Damage Modelling of Quasi-Brittle Materials:formulation, Valilotions and Numerical Implantations. Lille: Lille:Universite, des Sciences et Technologies de Lille.

Zhu, Q., Kondo, D., Shao, J., and Pensee, V. (2008). Micromechanical Modelling of Anisotropic Damage in Brittle Rocks and Application. Int. J. Rock Mech. Mining Sci.45, 467-477. doi:10.1016/j.ijrmms.2007.07.014

Conflict of Interest: Authors JH, HS, and DL were employed by the company Henan Geological and Mineral Resources Construction Engineering (Group) Co. Ltd.

The remaining authors declare that the research was conducted in the absence of any commercial or financial relationships that could be construed as a potential conflict of interest.

Publisher's Note: All claims expressed in this article are solely those of the authors and do not necessarily represent those of their affiliated organizations, or those of the publisher, the editors, and the reviewers. Any product that may be evaluated in this article, or claim that may be made by its manufacturer, is not guaranteed or endorsed by the publisher.

Copyright $\odot 2022 \mathrm{He}, \mathrm{Li}$, Jin, Wang, Zhang, Jia, Song and Liang. This is an openaccess article distributed under the terms of the Creative Commons Attribution License (CC BY). The use, distribution or reproduction in other forums is permitted, provided the original author(s) and the copyright owner(s) are credited and that the original publication in this journal is cited, in accordance with accepted academic practice. No use, distribution or reproduction is permitted which does not comply with these terms. 